\title{
VERIFICAÇÃO E ANÁLISE MORFOFUNCIONAL DAS CARACTERÍSTICAS DA MASTIGAÇÃO EM USUÁRIOS DE PRÓTESE DENTÁRIA REMOVÍVEL
}

\section{Verification and morfofunctional analysis of mastication characteristics in individuals using removable dental prosthesis}

\author{
Renata Veiga Andersen Cavalcanti (1), Esther Mandelbaum Gonçalves Bianchini (2)
}

\begin{abstract}
RESUMO
Objetivo: verificar as características da mastigação em indivíduos usuários de prótese dentária removível parcial e total, analisando prováveis fatores interferentes associados à função mastigatória. Métodos: participaram 53 indivíduos, ambos os sexos, entre 42 anos e 67 anos, divididos em dois grupos: 32 indivíduos usuários de prótese dentária total e/ou parcial removível estável (G1) e 21 indivíduos com dentição natural (G2). Foram critérios de exclusão: deficiência neurológica ou cognitiva, deformidades dentofaciais, disfunção temporomandibular e qualquer lesão bucal que pudesse interferir na mastigação. A avaliação foi realizada com pão de sal, solicitando-se mastigação habitual. Foram analisados: características das estruturas e da musculatura, tipo de corte, manutenção de vedamento labial, tempo de mastigação, número de ciclos mastigatórios, e tipo de mastigação: se uni ou bilateral, considerando-se predomínio unilateral em mais de $66 \%$ dos ciclos mastigatórios em um único lado. Resultados: foram verificadas alterações quanto à característica do músculo masseter e ao tipo de corte para G1 com diferenças estatisticamente significantes, quando comparado à G2. As demais estruturas orofaciais, lábios, língua, bochechas, mentual, e postura de lábios durante a mastigação apresentaram-se normais para ambos os grupos. Não houve diferença significante entre os grupos quanto aos movimentos mandibulares, ao tipo, tempo e número de ciclos mastigatórios e caracterização da deglutição. Conclusão: a mastigação em usuários de prótese dentária removível caracteriza-se por alterações no corte do alimento, indefinição de tipo mastigatório, vedamento labial satisfatório, média do tempo e de ciclos mastigatórios semelhantes àqueles obtidos para indivíduos com dentição natural.
\end{abstract}

DESCRITORES: Prótese Dentária; Prótese Parcial Removível; Mastigação

\section{INTRODUÇÃO}

A alta incidência de perdas dentárias é uma realidade que a população brasileira enfrenta apesar de todo avanço da odontologia. A perda dentária altera a homeostase do sistema estomatognático devido à modificação de parte do esqueleto facial, associado à perda de osso alveolar e resposta neuromuscular, interferindo na realização das funções

(1) Fonoaudióloga; Mestre em Fonoaudiologia pela Universidade Veiga de Almeida - RJ.

(2) Fonoaudióloga; Professora Adjunta do Mestrado Profissional em Fonoaudiologia da Universidade Veiga de Almeida RJ; Doutora em Ciências: Fisiopatologia Experimental pela Faculdade de Medicina da Universidade de São Paulo. de mastigação, deglutição, fala. As próteses dentárias retratam a possibilidade de melhora na realização dessas funções e do restabelecimento da estética, porém a adaptação das próteses requer cuidados, uma vez que a modificação morfofuncional pode dificultar a acomodação e estabilidade, principalmente nos casos de próteses totais ${ }^{1,2}$.

O edentulismo afeta os aspectos motores e sensoriais do processo mastigatório. A atrofia muscular, principalmente dos músculos masseteres, parece associar-se a uma perda do feedback sensorial periodontal com implicações na eficiência mastigatória na população de usuários de próteses. A redução perceptual e conseqüente informação neurosensorial parcial ou imprecisa dificultam a 
organização do padrão mastigatório uma vez que a textura dos alimentos não é percebida com a mesma precisão que a população dentada. Assim, a organização dos ciclos mastigatórios em relação ao bolo alimentar é consideravelmente reduzida para os usuários de próteses totais ${ }^{2,3}$.

Estudos ${ }^{3-8}$ ressaltam que a eficiência e/ou performance mastigatória em usuários de prótese é significativamente reduzida quando comparada a indivíduos com dentição natural. Sugerem, portanto, que as próteses totais forneceriam substituições funcionais pobres em relação às dentições completas.

O tempo de uso das próteses é também um aspecto relevante. Estudo ${ }^{7}$ relata que indivíduos, com tempo de uso de prótese maior que seis meses, têm melhor performance mastigatória, sugerindo que a adaptação do paciente com a prótese pode influenciar significantemente na sua performance mastigatória 7 . Outros autores ${ }^{4,9}$ descrevem um tempo menor de uso da prótese para que ocorra tal adaptação funcional. Porém, a adaptação e estabilidade parecem relacionar-se tanto à técnica de reabilitação odontológica quanto à situação miofuncional orofacial, podendo essa última ser considerada uma das causas insucesso na adaptação ou de instabilidade das próteses totais ${ }^{1,4,7}$.

Uma vez que a expectativa em melhorar o processo mastigatório, apresenta-se como fator determinante para a escolha de usar ou não a prótese dentária ${ }^{7-9}$, a integração dos trabalhos da Odontologia e da Fonoaudiologia pode auxiliar o paciente na adaptação às próteses e na reabilitação das suas funções orais dentro das limitações encontradas, favorecendo melhor satisfação e qualidade de vida para o indivíduo ${ }^{10}$, visto que, tanto a adaptação da prótese pode ficar comprometida por alterações miofuncionais, quanto às funções estomatognáticas podem estar modificadas devido à condição e tipo de prótese dentária ${ }^{11}$.

O objetivo desta pesquisa foi verificar as características da mastigação em indivíduos adultos, usuários de prótese dentária removível parcial e total, quanto ao: corte do alimento, tipo mastigatório se uni ou bilateral, tempo e número de ciclos mastigatórios, assim como a caracterização das estruturas orofaciais e deglutição; analisando prováveis fatores interferentes associados à função mastigatória.

\section{MÉTODOS}

Trata-se de pesquisa exploratória de corte transversal, realizada após processos éticos pertinentes, sendo considerada sem risco e com necessidade de Termo de Consentimento Livre e Esclarecido.
Fizeram parte, 53 indivíduos de ambos os sexos, com idade entre 42 anos e 67 anos, divididos em dois grupos: (G1) 32 indivíduos usuários de prótese dentária total e/ou parcial removível e (G2) 21 indivíduos não usuários de prótese dentária.

Foram excluídos dessa pesquisa os indivíduos que apresentaram: quaisquer déficits neurológicos e cognitivos; deformidades dentofaciais; sintoma de dor e desconforto na região temporomandibular; alterações ou dor em dentes e/ou periodonto; presença de rebordos edêntulos; presença de algum tipo de patologia ou lesões bucais que pudessem interferir na mastigação; menos que 20 dentes naturais ou substituídos por prótese; ausência de elemento dentário (natural ou prótese) ocasionando falha de contato dentário posterior e/ou anterior; e aqueles que tenham sido submetidos a tratamento fonoaudiológico.

O Grupo de pesquisa (G1) foi constituído por indivíduos provenientes da Clínica de Odontologia da Universidade Veiga de Almeida (UVA) e da Policlínica de Referência do Centro Universitário Vila Velha (UVV), sendo obedecidos os seguintes critérios de inclusão: tempo mínimo de uso das próteses de 3 meses ${ }^{4,9}$; tempo máximo de uso das próteses de 5 anos ${ }^{5}$ e que apresentassem estabilidade das próteses: relatadas pelo paciente como estável e sem queixas específicas.

O Grupo controle foi constituído por 21 indivíduos com dentição natural, obedecendo aos critérios de exclusão, balanceados quanto à faixa etária e gênero em relação ao grupo de pesquisa, que voluntariamente concordaram em participar dessa pesquisa.

Os materiais utilizados foram: termo de consentimento livre e esclarecido; avaliação de seleção com base nos critérios de exclusão e inclusão; protocolo de avaliação miofuncional orofacial para usuários de próteses dentárias ${ }^{12}$; protocolo de levantamento de dados desenvolvido para essa pesquisa; filmadora digital SONY DCR-SR100; DVD-R/CD-R; paquímetro digital 150/0,01mm PANTEC modelo 11108-150-1; cronômetro CRONOBIO SW2018; luvas; e pão de sal.

Foi aplicada, pela pesquisadora responsável, avaliação de seleção, constando de entrevista com o participante, avaliação clínica por meio de observação do estado geral do paciente e das estruturas do sistema estomatognático. Os indivíduos selecionados, conforme critérios de exclusão e inclusão, foram avaliados individualmente, utilizando-se o protocolo de avaliação miofuncional para usuários de próteses dentárias ${ }^{12}$, documentado em foto e vídeo seguindo-se protocolo de documentação fonoaudiológica miofuncional orofacial. Os registros em foto foram realizados: em foto de frente e perfil, 
tanto para face quanto para oclusão, e em vídeo durante a realização da mastigação para possibilitar análise e verificação posterior para checagem e confirmação dos dados.

A avaliação das estruturas do sistema estomatognático foi realizada por meio de avaliação clínica constando de observação, palpação e medição com paquímetro. Para a avaliação da função mastigatória foi utilizado pão de sal e solicitado ao participante que mastigasse cinco porções de forma habitual. Foi desprezada a primeira porção para análise, uma vez que o participante poderia direcionar a mastigação de forma atenta e controlada por estar em início de situação de avaliação e filmagem. Nas quatro porções seguintes foram anotados tempo e número de ciclos de cada porção e realizada a média em relação a cada uma dessas variáveis. Os dados foram registrados no protocolo de levantamento de dados especialmente voltado para os itens de interesse do presente estudo, sendo esses tabulados e posteriormente analisados (Figura 1).

As características levantadas foram classificadas da seguinte forma para posterior tratamento estatístico:

\section{I - Caracterização da prótese}

Para G1 as próteses foram classificadas quanto ao tipo como: Prótese Total Removível Superior, Prótese Total Removível Inferior, Prótese Parcial Removível Superior e Prótese Parcial Removível Inferior; e descritas quanto ao tempo de uso da prótese atual em meses, considerando-se desde o início da adaptação.

\section{II - Dados da avaliação}

A - Caracterização das estruturas orofaciais

Os lábios foram classificados como alterados quando se observou hipotonia ou hipertonia funcional, a partir da observação da postura habitual e solicitação de força contra resistência dos dedos do examinador. Foram classificados como normais quando apresentaram postura habitual de boca fechada sem esforço e presença de força e facilidade em executar a prova.

As bochechas foram classificadas como alteradas quando se observou hipotonia ou hipertonia funcional, a partir de solicitação de força do músculo bucinador contra resistência do dedo indicador do examinador. Foram classificadas como normais quando foi observada presença de força e facilidade em executar a prova.

A língua foi classificada como alterada quando se observou postura anteriorizada: apoio anterior ou interdental. Foi classificada como normal quando tais situações não foram observadas.
O músculo mentual foi classificado como alterado quando, em repouso, foi visualmente constatada a contração da musculatura, definido como funcionalmente hipertônico; e normal quando, em repouso, não foi constatada visualmente a contração da musculatura.

Masseteres: foi solicitado ao participante que fizesse um aperto de dentes para verificação perceptual da contração muscular por meio de toque com a ponta dos dedos indicador e médio do examinador sobre os músculos, bilateralmente. Foi classificado como alterado, quando constatada contração assimétrica, assinalando-se o lado de melhor desempenho no protocolo de levantamento de dados; e normal, quando a contração exercida pela musculatura foi considerada presente em ambos os lados e simétrica.

\section{B - Caracterização dos Movimentos Mandibulares}

Abertura e fechamento: foi verificada a amplitude, desvios no percurso e presença de ruídos articulares perceptíveis à inspeção clínica. Foi classificado como alterado, quando houve presença de desvios e/ou ruídos, sendo assinalado o lado do desvio.

Abertura máxima: foi solicitado ao participante para forjar um bocejo e manter a boca aberta em sua amplitude máxima. Utilizando o paquímetro, essa amplitude foi medida considerando-se a distância obtida entre os incisivos superiores e inferiores, somando-se a sobremordida. Foi utilizado como referência o intervalo entre $40 \mathrm{~mm}$ e $55 \mathrm{~mm}{ }^{13}$. Foi classificada como alterada quando se constatou amplitude reduzida ou aumentada em relação aos valores de referência.

Lateralidade Direita e Esquerda: inicialmente foi verificada a linha média dentária, observandose sua centralização. Em caso de discrepância foi medido o desvio de linha média, sendo esse considerado para o cálculo na obtenção da lateralidade 13. Para a verificação da amplitude de lateralidade do movimento mandibular, foi solicitada lateralização mandibular máxima, usando como referência o contato dental, e medida a distância entre a linha média superior e inferior, separadamente para o lado direito e para o lado esquerdo. Foi utilizado como referência o intervalo entre $7 \mathrm{~mm}$ a $11 \mathrm{~mm}^{13}$. Foi classificado como alterado quando constatada amplitude reduzida ou aumentada em relação aos valores de referência.

Protrusão: foi verificada a protrusão máxima medindo-se a distância entre as faces vestibulares do incisivo central superior e incisivo central inferior, com o paquímetro, na altura do plano oclusal, com o movimento mandibular protrusivo, mantendo como 
Identificação do sujeito:

I - Caracterização da amostra

Idade: .......anos Gênero: ( ) Masc. ( ) Fem.

II - Caracterização da prótese (apenas para G1):

Tipo: ( ) Prótese Total Removível Superior

( ) Prótese Total Removível Inferior

( ) Prótese Parcial Removível Superior

( ) Prótese Parcial Removível Inferior

Tempo de uso da prótese atual:

III - Dados da avaliação

A - Caracterização das estruturas

Lábios: () funcionalmente normais

Bochechas: ( ) funcionalmente normais

Língua: ( ) com postura anteriorizada

Mentual: ( ) funcionalmente normal

( ) funcionalmente hipotônicos ( ) funcionalmente hipertônicos

( ) funcionalmente hipotônicos ( ) funcionalmente hipertônicos

Masseteres:

( ) sem postura anteriorizada

B - Movimentos Mandibulares
() Contração assimétrica ( ) direito melhor

Abertura:

( )desvio para Direita

( ) limitações ( ) ruídos

( ) desvio para Esquerda

Fechamento: ( )desvio para Direita

( ) dor ( ) nada digno de nota (ndn)
( )desvio para Esquerda

( ) limitações
Abertura máxima: medida:

( ) ruídos

(Referência: 40 a $55 \mathrm{~mm})^{13}$

( ) dor ( ) nada digno de nota (ndn)

Lateralidade E: medida: .......mm ( ) aumentada ( ) diminuída ( ) amplitude normal

Lateralidade D: medida: ........mm ( ) aumentada ( ) diminuída ( ) amplitude normal

Protrusão: medida:

...mm ( ) aumentada

( ) com desvio

( ) diminuída ( ) amplitude normal

(Referência: 07 a $11 \mathrm{~mm})^{13}$

( ) sem desvio

C - Caracterização da Mastigação

- Tipo de corte: ( ) anterior ( ) lateral ( ) com a mão ( ) rasga

Postura dos lábios: ( ) abertos ( ) fechados

- Tempo de mastigação (cronometrado):

1aㅡ porção: seg

$2^{\mathrm{a}}$ porçãa: seg

3 e porção: seg

$4^{a}$ porção: seg

5a porção: _ـ__ seg

Média (2 a $5^{\underline{a}}$ porção):

- Número de ciclos:

1ª porção: ciclos

2a porção: — ciclos

3a porção: —— ciclos

4a porção: — ciclos

5a porção: ciclos

Média (2 $2^{\mathrm{a}}$ a $5^{\mathrm{a}}$ porção): seg

- Tipo de mastigação (número de ciclos realizados em cada lado)

1ㄹ porção:

$2^{\mathrm{a}}$ porção:

$3^{a}$ porção: ciclos lado direito e ciclos lado direito e

4⿳亠丷厂 porção: ciclos lado direito e ciclos lado esquerdo ciclos lado esquerdo

5a porção: ciclos lado direito e ciclos lado esquerdo ciclos lado esquerdo

Total de ciclos lado direito ( $2^{\mathrm{a}}$ a $5^{\mathrm{a}}$ porção): ciclos lado esquerdo

Total de ciclos lado esquerdo ( $2^{\mathrm{a}}$ a $5^{\mathrm{a}}$ porção):

( ) unilateral ( ) bilateral

D - Caracterização da deglutição do alimento mastigado

Com pressionamento: ( ) sim ( ) não

Movimento de cabeça: ( ) sim ( ) não

( ) Sem alterações significativas 
referência o contato dental posterior, somando-se a medida da sobressaliência. Foi utilizado como referência o intervalo entre $7 \mathrm{~mm}$ a $11 \mathrm{~mm}{ }^{13}$. Foi classificado como alterado quando constatada amplitude reduzida ou aumentada em relação aos valores de referência.

\section{C - Caracterização da Mastigação}

O tipo de corte foi classificado como: anterior, quando realizado com incisivos centrais e laterais; lateral, quando realizado pelos caninos e pré-molares ou quando realizado na região posterior pelos pré-molares e molares; com a mão, quando o participante partiu o alimento com as mãos antes de posicioná-lo entre os dentes, não ocorrendo a fase incisal da mastigação; ou rasgando o alimento, quando foi observado que o participante posicionou o alimento entre os dentes, mas não realizou a atividade de corte, utilizando os dentes como alavanca para rasgá-lo ${ }^{14,15}$. Foi classificado como alterado quando observado: corte lateral, rasga e com a mão.

A postura dos lábios durante a mastigação foi classificada como alterada quando observou-se lábios abertos; e normal para manutenção de lábios fechados.

O tempo de mastigação, de cada uma das cinco porções solicitadas, foi cronometrado e registrado, acionando-se o cronômetro no início da fase incisal e desligado quando se iniciou a primeira deglutição, observada pela elevação da laringe ${ }^{14}$. O tempo tabulado foi a média dos valores obtidos para cada sujeito descartando-se a 1 a porção.

O número de ciclos foi contado e registrado o número de ciclos mastigatórios de cada porção, considerando-se desde o primeiro ciclo mastigatório até o início da primeira deglutição. O número de ciclos tabulado foi a média dos valores obtidos para cada sujeito descartando-se a $1^{\underline{a}}$ porção.

O tipo de mastigação foi classificado como unilateral ou bilateral. Para essa classificação, foi contabilizado o número de ciclos realizados em cada lado (direito e esquerdo). A mastigação foi considerada como unilateral quando houve mais de $66 \%$ dos ciclos mastigatórios em um mesmo lado, e bilateral quando apresentou menos que $66 \%$ dos ciclos mastigatórios no total das 4 porções ${ }^{14}$.

\section{D - Caracterização da deglutição do alimento mastigado}

Com pressionamento: foi classificado como alterado quando se observou, no momento da deglutição, pressionamento de lábio e/ou língua.

Movimento de cabeça: foi classificado como alterado, quando se observou, no momento da deglutição, movimento associado da cabeça.
A deglutição foi classificada como alterada, quando se observou alteração em um ou nos dois itens anteriores; e normal, quando tais itens não foram observados.

Esta pesquisa iniciou-se após aprovação pela Comissão de Ética em Pesquisa da Universidade Veiga de Almeida, sob o número 79/07.

Para a análise estatística foram utilizados os softwares: SPSS V11.5, Minitab 14 e Excel XP, obedecendo-se aos seguintes critérios: análise estatística descritiva constando de cálculo das medidas-resumo, quanto às variáveis paramétricas (quantitativas), média aritmética simples, desvio-padrão, intervalo de confiança, valores mínimo e máximo; e variáveis não-paramétricas (qualitativas), tabela de freqüência absoluta e freqüência relativa (percentual).

A análise estatística e analítica para a comparação dos resultados entre G1 e G2 foi realizada por meio dos seguintes testes: Mann-Whitney, Teste de Igualdade de Duas Proporções, Intervalo de Confiança para Média. Para a avaliação do grau de relação entre as variáveis de cada grupo foi realizada Correlação de Spearman, Teste de Correlação e Teste Qui-Quadrado para Independência.

Foi definido para este trabalho um nível de significância de 0,05 (5\%), e todos os intervalos de confiança foram construídos com $95 \%$ de confiança estatística.

\section{RESULTADOS}

Foram selecionados 165 (100\%) prontuários de pacientes dos referidos ambulatórios que inicialmente enquadraram-se nos objetivos da pesquisa. Desses prontuários, $53(32,1 \%)$ pacientes não foram localizados ou não responderam ao contato; $21(12,7 \%)$ pacientes não aceitaram participar da pesquisa; $26(15,8 \%)$ foram excluídos pelos critérios da pesquisa; 18 (10,9\%) eram usuários de prótese fixa, e $15(9,1 \%)$ não terminaram o tratamento odontológico ou faltaram nas datas agendadas. Constitui-se assim a amostra para o presente trabaIho com 32 sujeitos.

$\mathrm{Na}$ caracterização dos grupos em relação à idade, G1 apresentou média de 55,31 anos e G2 média de 51,48 anos. A análise estatística através do Teste de Mann-Whitney apresentou p-valor de 0,066, mostrando diferença estatisticamente não significante, ou seja, os grupos são homogêneos quanto a variável idade, confirmando que a variável foi controlada.

$\mathrm{Na}$ caracterização dos grupos em relação ao sexo, G1 apresentou 24 (75\%) dos sujeitos do sexo feminino e oito (25\%) do masculino; G2 apresentou $12(57,1 \%)$ dos sujeitos do sexo feminino e nove 
(42,9\%) do masculino. A análise estatística através do Teste de igualdade de duas proporções apresentou $p$-valor de 0,173 , mostrando diferença estatisticamente não significante entre os grupos, confirmando que a variável foi controlada.

A Tabela 1 mostra os resultados quanto à caracterização do tipo de prótese e sua localização. Em relação às próteses superiores, observou-se predomínio de próteses totais $(56,3 \%)$ em relação ao percentual de próteses parciais $(34,4 \%)$, porém a análise estatística expressa quanto aos p-valores das comparações entre os tipos de próteses, mostrou diferença estatisticamente não significante $(p$-valor 0,079$)$. Referente às próteses inferiores, o percentual mais prevalente foi de próteses parciais $(50,0 \%)$ quando comparado à presença de próteses totais e ausência de prótese, com diferenças estatisticamente significantes ( $p$-valor 0,039).

Quanto à distribuição dos participantes de G1 referente ao tempo de uso das próteses, observouse média de tempo de uso das próteses superiores de 31,6 meses; e de 33,3 meses para uso de próteses inferiores.

Tabela 1 - Distribuição absoluta e relativa dos participantes de G1 quanto ao tipo de prótese e localização

\begin{tabular}{ccccc}
\hline \multirow{2}{*}{ Prótese } & \multicolumn{2}{c}{ PS } & \multicolumn{2}{c}{ PI } \\
\cline { 2 - 5 } & Qtde & $\%$ & Qtde & $\%$ \\
\hline Nenhuma & 3 & $9,4 \%$ & 8 & $25,0 \%$ \\
PPR & 11 & $34,4 \%$ & 16 & $50,0 \%$ \\
PT & 18 & $56,3 \%$ & 8 & $25,0 \%$ \\
\hline
\end{tabular}

Prótese Parcial Removível (PPR) e Prótese Total (PT), Prótese Superior (PS) e Prótese Inferior (PI).

A Tabela 2 mostra a caracterização das estruturas orofaciais, dos movimentos mandibulares, do corte do alimento, do tipo mastigatório e da deglutição, analisados para os 2 grupos em questão. Verificou-se somente quanto ao músculo masseter a existência de diferença estatisticamente significante entre os grupos, onde G1 apresentou maior porcentagem de sujeitos com alteração. Na caracterização do corte do alimento, verifica-se que G1 possui maior percentual de participantes que apresentam corte alterado do que G2, com diferenças estatisticamente significantes. Na caracterização do tipo mastigatório verifica-se que, embora exista pequena diferença dos percentuais entre os grupos, as mesmas não são consideradas estatisticamente significantes.
A Tabela 3 mostra a caracterização dos participantes quanto às variáveis tempo e número de ciclos mastigatórios durante a mastigação. Verificase que, embora existam mínimas diferenças entre os grupos para as duas variáveis, as mesmas não são estatisticamente significantes.

Visando a avaliação do grau de relação entre as variáveis de cada grupo, foram realizados alguns cruzamentos de dados.

A Tabela 4 mostra o grau de relação entre as variáveis: tempo de uso da prótese, alterações das estruturas orofaciais e caracterização da mastigação para G1. Verificou-se correlação estatística somente entre bochecha e tempo de uso da prótese superior, indicando que os participantes com menor tempo de uso de prótese superior são os que mais apresentam alterações de bochecha. Quanto às características da mastigação e tempo de uso da prótese, verificou-se correlação positiva somente entre alteração do corte e tempo de uso da prótese. Apesar de observar-se correlação fraca, a relação significante pode indicar que à medida que o tempo de uso de prótese aumenta, maior o número de sujeitos com alteração no corte.

A Tabela 5 mostra o grau de relação entre as variáveis: características da mastigação, e entre idade e mastigação. Quanto às características da mastigação, verifica-se correlação positiva entre tempo e número de ciclos mastigatórios com relação estatisticamente significante para G1 e também para G2, ou seja, quanto maior o tempo mastigatório maior o número de ciclos. Quanto à idade e mastigação, verificou-se correlação referente ao número de ciclos com relação estatisticamente significante, somente para G2. A correlação regular pode indicar um aumento do número de ciclos mastigatórios quanto maior a idade.

A Tabela 6 mostra a relação entre as variáveis: caracterização dos movimentos mandibulares quanto à lateralidade e tipo mastigatório. Não foram encontradas correlações entre essas variáveis para ambos os grupos, sugerindo que a amplitude dos movimentos mandibulares dirigidos não interfere no tipo de mastigação.

Foram também realizados cruzamentos de dados entre as variáveis: características das estruturas orofaciais, tempo mastigatório e número de ciclos; movimentos mandibulares e tempo de uso da prótese; e entre as características da mastigação e da deglutição. Não foram constatadas correlações entre tais variáveis. 
Tabela 2 - Caracterização das estruturas orofaciais, dos movimentos mandibulares, do corte do alimento, do tipo mastigatório e da deglutição para os 2 grupos analisados

\begin{tabular}{|c|c|c|c|c|c|c|c|}
\hline & & & \multicolumn{2}{|c|}{ G1 } & \multicolumn{2}{|c|}{ G2 } & \multirow{2}{*}{ p-valor* } \\
\hline & & & Qtde & $\%$ & Qtde & $\%$ & \\
\hline \multirow{10}{*}{$\begin{array}{l}\text { Estruturas } \\
\text { orofaciais }\end{array}$} & \multirow{2}{*}{ Lábios } & Alterado & 3 & $9,4 \%$ & 0 & $0,0 \%$ & \multirow{2}{*}{0,149} \\
\hline & & Normal & 29 & $90,6 \%$ & 21 & $100 \%$ & \\
\hline & \multirow{2}{*}{ Bochechas } & Alterado & 5 & $15,6 \%$ & 1 & $4,8 \%$ & \multirow{2}{*}{0,222} \\
\hline & & Normal & 27 & $84,4 \%$ & 20 & $95,2 \%$ & \\
\hline & \multirow{2}{*}{ Língua } & Alterado & 4 & $12,5 \%$ & 0 & $0,0 \%$ & \multirow{2}{*}{0,092} \\
\hline & & Normal & 28 & $87,5 \%$ & 21 & $100 \%$ & \\
\hline & \multirow{2}{*}{ Mentual } & Alterado & 5 & $15,6 \%$ & 1 & $4,8 \%$ & \multirow{2}{*}{0,222} \\
\hline & & Normal & 27 & $84,4 \%$ & 20 & $95,2 \%$ & \\
\hline & \multirow{2}{*}{ Masseter } & Alterado & 6 & $18,8 \%$ & 0 & $0,0 \%$ & \multirow{2}{*}{$0,035^{\star *}$} \\
\hline & & Normal & 26 & $81,3 \%$ & 21 & $100 \%$ & \\
\hline \multirow{12}{*}{$\begin{array}{l}\text { Movimentos } \\
\text { mandibulares }\end{array}$} & \multirow{2}{*}{ Abertura } & Alterado & 12 & $37,5 \%$ & 11 & $52,4 \%$ & \multirow{2}{*}{0,285} \\
\hline & & Normal & 20 & $62,5 \%$ & 10 & $48 \%$ & \\
\hline & \multirow{2}{*}{ fechamento } & Alterado & 10 & $31,3 \%$ & 4 & $19,0 \%$ & \multirow{2}{*}{0,324} \\
\hline & & Normal & 22 & $68,8 \%$ & 17 & $81 \%$ & \\
\hline & \multirow{2}{*}{$\begin{array}{l}\text { abertura } \\
\text { máxima }\end{array}$} & Alterado & 8 & $25,0 \%$ & 4 & $19,0 \%$ & \multirow{2}{*}{0,613} \\
\hline & & Normal & 24 & $75,0 \%$ & 17 & $81 \%$ & \\
\hline & \multirow{2}{*}{$\begin{array}{c}\text { lateralidade } \\
\text { esquerda }\end{array}$} & Alterado & 16 & $50,0 \%$ & 7 & $33,3 \%$ & \multirow{2}{*}{0,231} \\
\hline & & Normal & 16 & $50,0 \%$ & 14 & $67 \%$ & \\
\hline & \multirow{2}{*}{$\begin{array}{l}\text { lateralidade } \\
\text { direita }\end{array}$} & Alterado & 17 & $53,1 \%$ & 9 & $42,9 \%$ & \multirow{2}{*}{0,465} \\
\hline & & Normal & 15 & $46,9 \%$ & 12 & $57 \%$ & \\
\hline & \multirow{2}{*}{$\begin{array}{c}\text { Protrusão } \\
\text { Abertura }\end{array}$} & Alterado & 17 & $53,1 \%$ & 7 & $33,3 \%$ & \multirow{2}{*}{0,157} \\
\hline & & Normal & 15 & $46,9 \%$ & 14 & $67 \%$ & \\
\hline \multirow{4}{*}{ Mastigação } & \multirow{2}{*}{$\begin{array}{l}\text { Corte do } \\
\text { alimento }\end{array}$} & Alterado & 23 & $71,9 \%$ & 2 & $9,5 \%$ & \multirow{2}{*}{$<0,001^{* *}$} \\
\hline & & Normal & 9 & $28,1 \%$ & 19 & $90 \%$ & \\
\hline & Tipo & Bilateral & 15 & $46,9 \%$ & 12 & $57,1 \%$ & 0165 \\
\hline & mastigatório & Unilateral & 17 & $53,1 \%$ & 9 & $43 \%$ & 0,400 \\
\hline Dealuticão & & Alterado & 21 & $65,6 \%$ & 12 & $57,1 \%$ & 0533 \\
\hline Degiutiçao & & Normal & 11 & $34,4 \%$ & 9 & $43 \%$ & 0,533 \\
\hline
\end{tabular}

* Teste de Igualdade de duas proporções

** Diferença estatisticamente significante

Tabela 2 - Caracterização das estruturas orofaciais, dos movimentos mandibulares, do corte do alimento, do tipo mastigatório e da deglutição para os 2 grupos analisados

\begin{tabular}{lcccc}
\hline \multirow{2}{*}{ Mastigação } & \multicolumn{2}{c}{ tempo (seg.) } & número de ciclos & G2 \\
\cline { 2 - 5 } & G1 & G2 & G1 & 21,43 \\
Média & 22,07 & 19,06 & 22,06 & 21,5 \\
Mediana & 21,64 & 19,84 & 19,25 & 6,73 \\
Desvio Padrão & 7,47 & 4,94 & 10,65 & 21 \\
N & 32 & 21 & 32 & 2,88 \\
Intervalo de Confiança & 2,59 & 2,11 & 3,69 & 0,750 \\
\hline p-valor* & & 0,237 & & \\
\hline
\end{tabular}

* Teste de Igualdade de duas proporções

** Diferença estatisticamente significante 
Tabela 3 - Comparação dos grupos para tempo e número de ciclos mastigatórios

\begin{tabular}{lcccc}
\hline \multirow{2}{*}{ Mastigação } & \multicolumn{3}{c}{ tempo (seg.) } & número de ciclos \\
\cline { 2 - 5 } & G1 & G2 & G1 & G2 \\
\hline Média & 22,07 & 19,06 & 22,06 & 21,43 \\
Mediana & 21,64 & 19,84 & 19,25 & 21,5 \\
Desvio Padrão & 7,47 & 4,94 & 10,65 & 6,73 \\
N & 32 & 21 & 32 & 21 \\
Intervalo de Confiança & 2,59 & 2,11 & 3,69 & 2,88 \\
\hline p-valor* & \multicolumn{3}{c}{0,750} \\
\hline
\end{tabular}

* Teste de Mann-Whitney

Tabela 4 - Grau de relação entre as variáveis: estruturas orofaciais e tempo de uso da prótese e entre as características da mastigação e tempo de uso da prótese

\begin{tabular}{|c|c|c|c|c|}
\hline & Par de variáveis & Prótese & Correlação* & p-valor ${ }^{* *}$ \\
\hline \multirow{10}{*}{$\begin{array}{l}\text { Estruturas orofaciais e } \\
\text { tempo de uso da prótese }\end{array}$} & \multirow{2}{*}{$\begin{array}{l}\text { Lábios } \mathrm{x} \text { tempo de uso } \\
\text { da prótese }\end{array}$} & OS & $-19,9 \%$ & 0,275 \\
\hline & & $\mathrm{PI}$ & $-9,4 \%$ & 0,609 \\
\hline & \multirow{2}{*}{$\begin{array}{c}\text { Bochechas } x \text { tempo de uso } \\
\text { da prótese }\end{array}$} & OS & $-43,2 \%$ & $0,013^{\star \star *}$ \\
\hline & & $\mathrm{PI}$ & $2,4 \%$ & 0,898 \\
\hline & \multirow{2}{*}{$\begin{array}{c}\text { Língua } x \text { tempo de uso } \\
\text { da prótese }\end{array}$} & OS & $-28,9 \%$ & 0,109 \\
\hline & & $\mathrm{PI}$ & $-14,5 \%$ & 0,428 \\
\hline & \multirow{2}{*}{$\begin{array}{c}\text { Mentual } x \text { tempo de uso } \\
\text { da prótese }\end{array}$} & OS & $-21,1 \%$ & 0,245 \\
\hline & & $\mathrm{PI}$ & $-14,6 \%$ & 0,424 \\
\hline & \multirow{2}{*}{$\begin{array}{l}\text { Masseter } \mathrm{x} \text { tempo de uso } \\
\text { da prótese }\end{array}$} & OS & $-0,4 \%$ & 0,981 \\
\hline & & $\mathrm{PI}$ & $-17,1 \%$ & 0,349 \\
\hline \multirow{8}{*}{$\begin{array}{l}\text { Características da } \\
\text { mastigação e tempo de } \\
\text { uso da prótese }\end{array}$} & \multirow{2}{*}{$\begin{array}{l}\text { Corte } \mathrm{x} \text { tempo de uso } \\
\text { da prótese }\end{array}$} & OS & $36,8 \%$ & $0,038^{\star \star \star}$ \\
\hline & & $\mathrm{PI}$ & $30,9 \%$ & 0,086 \\
\hline & \multirow{2}{*}{$\begin{array}{l}\text { Tempo mastigatório } \mathrm{x} \\
\text { tempo de uso da prótese }\end{array}$} & OS & $7,1 \%$ & 0,698 \\
\hline & & $\mathrm{PI}$ & $-5,7 \%$ & 0,757 \\
\hline & \multirow{2}{*}{$\begin{array}{l}\text { Ciclos mastigatórios } x \\
\text { tempo de uso da prótese }\end{array}$} & OS & $-5,5 \%$ & 0,763 \\
\hline & & $\mathrm{PI}$ & $-10,2 \%$ & 0,580 \\
\hline & \multirow{2}{*}{$\begin{array}{l}\text { Tipo mastigatório } x \\
\text { tempo de uso da prótese }\end{array}$} & OS & $-5,8 \%$ & 0,752 \\
\hline & & $\mathrm{PI}$ & $15,4 \%$ & 0,399 \\
\hline
\end{tabular}

Prótese Superior (PS) e Prótese Inferior (PI)

* Correlação de Spearman

** Teste de Correlação

*** Diferença estatisticamente significante

Tabela 5 - Grau de relação entre as variáveis: características da mastigação, e entre idade e mastigação

\begin{tabular}{|c|c|c|c|c|}
\hline & Par de variáveis & GRUPO & Correlação* & p-valor** \\
\hline \multirow{6}{*}{$\begin{array}{l}\text { Características da } \\
\text { mastigação }\end{array}$} & \multirow{2}{*}{$\begin{array}{l}\text { Tempo mastigatório x } \\
\text { número de ciclos }\end{array}$} & G1 & $82,5 \%$ & $<0,001^{\star \star \star}$ \\
\hline & & G2 & $83,0 \%$ & $<0,001^{\star * *}$ \\
\hline & \multirow{2}{*}{$\begin{array}{l}\text { Tempo mastigatório } \mathrm{x} \\
\text { tipo mastigatório }\end{array}$} & G1 & $-0,3 \%$ & 0,985 \\
\hline & & G2 & $15,9 \%$ & 0,491 \\
\hline & \multirow{2}{*}{$\begin{array}{l}\text { Número de ciclos } x \\
\text { tipo mastigatório }\end{array}$} & G1 & $-1,4 \%$ & 0,941 \\
\hline & & G2 & $39,8 \%$ & 0,074 \\
\hline \multirow{8}{*}{ Idade e mastigação } & \multirow{2}{*}{ Idade $x$ corte } & G1 & $-3,4 \%$ & 0,854 \\
\hline & & G2 & $-13,5 \%$ & 0,561 \\
\hline & \multirow{2}{*}{ Idade $\mathrm{x}$ tempo mastigatório } & G1 & $9,2 \%$ & 0,615 \\
\hline & & G2 & $21,6 \%$ & 0,347 \\
\hline & \multirow{2}{*}{ Idade x número de ciclos } & G1 & $15,0 \%$ & 0,414 \\
\hline & & G2 & $43,9 \%$ & $0,047^{\star \star \star}$ \\
\hline & \multirow{2}{*}{ Idade $\mathrm{x}$ tipo mastigatório } & G1 & $-15,6 \%$ & 0,393 \\
\hline & & G2 & $33,5 \%$ & 0,137 \\
\hline
\end{tabular}

* Correlação de Spearman

** Teste de Correlação 
Tabela 6 - Relação entre as variáveis: lateralidade mandibular e tipo mastigatório

\begin{tabular}{|c|c|c|c|c|c|c|c|c|c|c|c|}
\hline & & & \multicolumn{2}{|c|}{ Aumentada } & \multicolumn{2}{|c|}{ Diminuída } & \multicolumn{2}{|c|}{ Normal } & \multicolumn{2}{|c|}{ Total } & \multirow{2}{*}{ p-valor } \\
\hline & & & Qtde & $\%$ & Qtde & $\%$ & Qtde & $\%$ & Qtde & $\%$ & \\
\hline \multirow{8}{*}{ 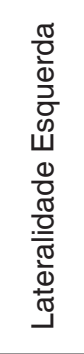 } & \multirow{4}{*}{ G1 } & bilateral & 0 & $0 \%$ & 6 & $42,9 \%$ & 9 & $56,3 \%$ & 15 & $46,9 \%$ & \multirow{4}{*}{0,300} \\
\hline & & unilateral D & 1 & $50 \%$ & 1 & $7,1 \%$ & 3 & $18,8 \%$ & 5 & $15,6 \%$ & \\
\hline & & unilateral E & 1 & $50 \%$ & 7 & $50,0 \%$ & 4 & $25,0 \%$ & 12 & $37,5 \%$ & \\
\hline & & Total & 2 & $6,3 \%$ & 14 & $43,8 \%$ & 16 & $50,0 \%$ & 32 & $100 \%$ & \\
\hline & \multirow{4}{*}{ G2 } & bilateral & 1 & $50 \%$ & 3 & $60 \%$ & 8 & $57,1 \%$ & 12 & $57,1 \%$ & \multirow{4}{*}{0,928} \\
\hline & & unilateral D & 1 & $50 \%$ & 1 & $20 \%$ & 4 & $28,6 \%$ & 6 & $28,6 \%$ & \\
\hline & & unilateral E & 0 & $0 \%$ & 1 & $20 \%$ & 2 & $14,3 \%$ & 3 & $14,3 \%$ & \\
\hline & & Total & 2 & $9,5 \%$ & 5 & $23,8 \%$ & 14 & $66,7 \%$ & 21 & $100 \%$ & \\
\hline \multirow{8}{*}{ 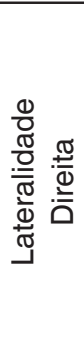 } & \multirow{4}{*}{ G1 } & bilateral & 2 & $50 \%$ & 6 & $46 \%$ & 7 & $47 \%$ & 15 & $46,9 \%$ & \multirow{4}{*}{0,521} \\
\hline & & unilateral D & 0 & $0 \%$ & 1 & $8 \%$ & 4 & $27 \%$ & 5 & $15,6 \%$ & \\
\hline & & unilateral E & 2 & $50 \%$ & 6 & $46 \%$ & 4 & $27 \%$ & 12 & $37,5 \%$ & \\
\hline & & Total & 4 & $12,5 \%$ & 13 & $40,6 \%$ & 15 & $46,9 \%$ & 32 & $100 \%$ & \\
\hline & \multirow{4}{*}{ G2 } & bilateral & 3 & $100 \%$ & 4 & $67 \%$ & 5 & $42 \%$ & 12 & $57,1 \%$ & \multirow{4}{*}{0,415} \\
\hline & & unilateral D & 0 & $0 \%$ & 1 & $17 \%$ & 5 & $42 \%$ & 6 & $28,6 \%$ & \\
\hline & & unilateral E & 0 & $0 \%$ & 1 & $17 \%$ & 2 & $17 \%$ & 3 & $14,3 \%$ & \\
\hline & & Total & 3 & $14,3 \%$ & 6 & $28,6 \%$ & 12 & $57,1 \%$ & 21 & $100 \%$ & \\
\hline
\end{tabular}

* Teste Qui-Quadrado

\section{DISCUSSÃO}

Para a realização desse estudo buscou-se homogeneidade entre os participantes dos grupos em relação à idade e gênero, controlando assim essas variáveis, uma vez que na literatura encontramos divergências quanto a interferência ou não dessas variáveis na função mastigatória ${ }^{16-18}$.

Para a avaliação funcional da mastigação foi escolhido o pão de sal por ser um alimento de consistência firme que favorece maior tempo de mastigação quando comparados a outros alimentos ${ }^{17}$. Esta padronização também foi importante para controlar esta variável, já que a consistência do alimento é referida como interferente no tempo mastigatório ${ }^{15,17,19}$.

Quanto aos tipos de próteses removíveis, verificou-se na amostra pesquisada prevalência de próteses totais no arco superior e de próteses parciais no arco inferior. Como, para essa pesquisa, o tipo de prótese, se total ou parcial, não foi controlado, não se buscou as associações quanto aos tipos utilizados e razão dessas escolhas.

Em relação às estruturas orofaciais, só foi observada diferença estatisticamente significante entre os percentuais de sujeitos dos grupos G1 e G2 referente às alterações do músculo masseter, onde os usuários de prótese (G1) apresentam maior porcentagem de sujeitos com alteração. Embora em número reduzido, esse achado concorda com estudo ${ }^{3}$ que indica que o edentulismo afeta as alterações dos aspectos motores e sensoriais do pro- cesso mastigatório, favorecendo a atrofia muscular, principalmente do masseter. Nas demais estruturas: lábios, bochechas, língua e mentual, não foram constatadas diferenças estatisticamente significantes, mas sim resultados semelhantes. Para ambos os grupos, observou-se maior porcentagem de sujeitos com estruturas normais indicando que a perda dos dentes seguida do uso de próteses, não determinou alterações nas estruturas orofaciais. Esse resultado não está de acordo com estudos ${ }^{1,11}$ que indicam que a perda dentária pode alterar a morfologia e a musculatura do sistema estomatognático. Vale ressaltar, que a diferença significante para o músculo masseter ocorreu, provavelmente, devido ao fato de que G2 não apresentou nenhum indivíduo com alteração. Analisando os resultados quanto à porcentagem descritiva, a diferença para masseter, clinicamente também parece não ser significante, uma vez que apenas $18,8 \%$ dos sujeitos de G1 apresentou alterações nesse músculo. Portanto, a hipótese inicialmente formulada de que as estruturas orofaciais de G1 encontrar-se-iam mais alteradas, quando comparadas a G2 não foi confirmada.

Ao analisar o comportamento dos participantes referente aos movimentos mandibulares observa-se que, em ambos os grupos existe importante percentual de sujeitos com movimentos de abertura alterados, sendo em maior número para G2. Quanto à lateralidade observou-se maior número de participantes com lateralidade e protrusão alterada para G1 quando comparado a G2, embora as diferenças 
não sejam estatisticamente significantes. O movimento mandibular de fechamento, assim como as amplitudes de abertura máxima apresentaram-se normais para a maioria dos sujeitos nos dois grupos estudados. Portanto, em relação aos movimentos mandibulares também não ocorreu diferença estatística entre os percentuais dos dois grupos, ou seja, o uso de próteses parece não interferir nesses movimentos, uma vez que os grupos comportam-se de maneira semelhante. Esses dados estão de acordo com outros estudos que comparam grupos de usuários de prótese e com dentição natural, ambos com queixas e sintomas de disfunções temporomandibulares, que mostram resultados similares entre os grupos quanto a movimentos mandibulares, e indicam que o uso de prótese não influencia tais problemas ${ }^{10,20,21}$. Concorda também com estudo ${ }^{22}$ que mostrou que não ocorrem mudanças significativas nos padrões de movimentos mandibulares pré e pós-inserção de próteses totais.

Quanto à avaliação da mastigação, foi encontrada diferença entre os grupos em relação ao corte do alimento, onde G1 apresenta mais indivíduos com alteração no corte do alimento que G2, perfazendo $71,9 \%$ e $9,5 \%$ respectivamente para cada grupo. Esse resultado pode ser decorrente da força de mordida apresentar-se diminuída nos usuários de prótese quando comparados a indivíduos com dentição natural como mostraram estudos anteriores ${ }^{4,6}$. Outra possibilidade a ser levantada referese à instabilidade de preensão do alimento devido ao fato da prótese ser removível. Embora todos os indivíduos da amostra tenham relatado estabilidade da prótese, esse dado não foi questionado em relação ao corte do alimento. Durante o corte, levando-se em consideração a maior força exigida e o posicionamento anterior do alimento, pode-se favorecer a instabilidade. Além disso, a simples insegurança dos indivíduos por usarem próteses removíveis, suscitaria receio em cortar os alimentos anteriormente.

Em relação ao tipo mastigatório observou-se distribuição semelhante para mastigação bilateral e para mastigação unilateral para os 2 grupos. Portanto, o padrão mastigatório quanto ao tipo, se bilateral ou unilateral, parece não se relacionar ao uso de prótese, na amostra estudada. O padrão mastigatório bilateral é citado na literatura como ideal e dependente da integridade das estruturas estomatognáticas ${ }^{15,23}$, porém estudo ${ }^{24}$ verificou que as más oclusões não direcionam o padrão unilateral mas favorecem os movimentos verticais. A textura do alimento poderia ser um fator influente na lateralidade ${ }^{25}$, porém essa variável foi controlada neste estudo, uma vez que só foi utilizado um tipo de alimento para a avaliação. Os resultados do presente estudo não corroboram com estudo ${ }^{1}$ que encontrou predominância do padrão unilateral logo após a instalação da PT e após um mês de uso da PT, sendo que a persistência do padrão foi associada à dificuldade de adaptação a nova prótese ou a um padrão já previamente estabelecido. Porém neste estudo ${ }^{1}$, só foram avaliados indivíduos desdentados totais, diferentemente do atual estudo. Existem ainda estudos ${ }^{24,26}$ que sugerem a existência de dominância cerebral quanto à lateralidade para a mastigação, porém essa variável não verificada no presente trabalho.

Em relação ao tempo e número de ciclos mastigatórios, embora exista diferença entre os grupos tanto para tempo quanto para ciclos, as mesmas não podem ser consideradas estatisticamente significantes. O valor do tempo mastigatório dos indivíduos usuários de prótese deste estudo está próximo ao valor encontrado em estudo ${ }^{17} \mathrm{com}$ indivíduos com dentição natural. Portanto, o uso de prótese parece não determinar variação específica quanto a tempo e ciclo mastigatório. Uma vez que nesse estudo foi utilizado apenas um tipo de alimento, os resultados obtidos não podem ser comparados a trabalhos que mostram que a consistência do alimento interfere no tempo mastigatório ${ }^{15,17,19}$. Apesar de não serem encontrados estudos avaliando o tempo e número de ciclos mastigatórios, e sim a eficiência mastigatória, esperava-se encontrar uma diferença significativa entre os grupos. A hipótese seria que os usuários de prótese tivessem o tempo e número de ciclos aumentados em relação aos indivíduos com dentição natural, pois se verificam estudos ${ }^{3-8}$ acerca de eficiência e/ou performance mastigatória, nos quais a eficiência é citada como significativamente reduzida nos usuários de prótese quando comparados aos indivíduos com dentição natural ${ }^{3-8}$. Entretanto essa hipótese não foi confirmada, podendo-se inferir que o tempo e número de ciclos mastigatórios talvez não reflitam eficiência mastigatória.

A caracterização da deglutição do alimento mastigado também não mostrou diferença estatisticamente significante entre os dois grupos. Esperavase diferença entre os grupos, já que existe estudo ${ }^{8}$ mostrando que a mastigação ineficiente nos usuários de prótese resultaria em um bolo alimentar menos fragmentado, o que favoreceria alteração na deglutição. Porém, no presente estudo, os grupos comportaram-se de maneira semelhante quanto a essa função, sugerindo que o uso de prótese parece não interferir na deglutição, corroborando com estudos ${ }^{1,2}$ que não observaram diferença significante na função de deglutição antes e após a inserção de próteses totais em indivíduos desdentados totais. 
Vale ressaltar que ao analisar a porcentagem descritiva dos resultados verificou-se que a presença de alteração da deglutição foi grande em ambos os grupos. Pode-se questionar se tal situação seria decorrente da mastigação, similar em ambos os grupos, ou à própria característica do alimento que, por ser seco, poderia dificultar a deglutição produzindo maior esforço. Por tratar-se de indivíduos adultos, não seria esperado maior número de sujeitos com alteração da deglutição e assim, esse resultado poderia relacionar-se à situação de avaliação, que talvez favoreça modificação dos padrões habituais do indivíduo. A análise do padrão de deglutição é bastante subjetiva em exame clínico e os critérios são bastante variados, além de ser conhecida a interferência de algumas variáveis que não foram controladas nesse estudo, tais como tipologia facial, volume de tonsilas palatinas, profundidade e largura do palato e tamanho da língua. Assim, sugere-se que novos estudos envolvendo deglutição sejam propostos para usuários de prótese dentária, com controle dessas variáveis e revisão da situação de avaliação subjetiva.

No presente estudo, buscou-se realizar também a análise dos grupos separadamente visando a verificação da correlação entre as variáveis do mesmo grupo. Analisando-se as estruturas orofaciais e tempo de uso da prótese foi verificada correlação entre alteração de bochecha e tempo de uso da prótese, indicando menor número de sujeitos com alteração na musculatura da bochecha à medida que aumenta o tempo de uso da prótese superior. De maneira correlata, embora não significantes, todas as outras correlações também foram inversas/negativas, o que pode sugerir a diminuição de alteração das estruturas orofaciais a medida que o tempo de uso da prótese aumenta, corroborando com estudos que mostram que para a adaptação funcional, é necessário pelo menos um mês de uso da prótese ${ }^{4}$, três meses ${ }^{9}$, ou mais que seis meses ${ }^{7}$.

A ausência de relação entre os movimentos mandibulares e o tempo de uso da prótese parece mostrar que o tempo de uso não interfere nos movimentos mandibulares, estando de acordo com alguns estudos anteriores ${ }^{10,20-22}$.

A presença ou não de alteração na amplitude dos movimentos mandibulares quanto à lateralidade, seja direita ou esquerda, parece não interferir no tipo mastigatório em ambos os grupos. Esse resultado já era o esperado, pois o tipo mastigatório encontrado foi semelhante para os dois grupos. Entretanto, a lateralidade aumentada para um dos lados, poderia facilitar o movimento mandibular utilizado na mastigação para esse mesmo lado, direcionando assim o lado de mastigação. De maneira correlata, observando-se lateralidade diminuída, seria esperada mastigação contra-lateral ou bilateral como padrão facilitador. Analisando-se os grupos separadamente, essa característica não foi verificada, obtendo-se predomínio de mastigação à direita ou à esquerda, independente do lado de restrição ou de maior lateralidade.

A relação entre as características da mastigação e tempo de uso da prótese só apresentou correlação estatisticamente significante entre corte do alimento e tempo de uso da prótese, indicando que quanto mais tempo de uso da prótese, maior o número de sujeitos com alteração no corte. Visando estudos posteriores, pode-se sugerir que essa situação relacione-se a possíveis desgastes do dente artificial, ou pelo receio do deslocamento da prótese na hora do corte.

Não houve relação entre tempo, ciclos e tipo mastigatório e o tempo de uso da prótese, indicando que próteses com tempo de uso entre dois e sessenta meses parecem não interferir nessas características mastigatórias.

Ao correlacionar as características da mastigação: tempo e número de ciclos mastigatórios, verificou-se relação direta para ambos os grupos, indicando que à medida que aumenta o tempo mastigatório aumenta o número de ciclos mastigatórios, e vice-versa, conforme esperado.

$\mathrm{Na}$ correlação entre as características da mastigação e a deglutição não se verificou relação estatisticamente significante, sugerindo que as características mastigatórias parecem não interferir na deglutição, discordando de alguns estudos anteriores ${ }^{2,6}$.

Foi realizada a correlação entre idade e mastigação por haver estudos anteriores ${ }^{16,18}$ que analisam estes dados. Buscou-se assim identificar mais um possível fator associado que foi encontrado somente para G2, em relação direta, o que pode sugerir que quanto maior a idade, maior o número de ciclos mastigatórios. Porém como o grau de relação obtido foi apenas regular (entre 40 e 60\%). Respeitando-se as limitações desse estudo quanto a essa variável, o achado para G2 corrobora com apenas com um dos estudos anteriores ${ }^{16}$. Sugere-se que novos estudos sejam propostos buscando-se verificar a interferência da faixa etária.

\section{CONCLUSÃO}

A partir da metodologia utilizada no presente trabalho, verificou-se que a mastigação em usuários de prótese dentária removível caracteriza-se por alterações no corte do alimento, indefinição de tipo mastigatório específico, observando-se padrão 
bilateral e unilateral, com postura de lábios fechados e valores médios do tempo e do número de ciclos mastigatórios compatíveis com dados de referência para indivíduos com dentição natural.

A caracterização das estruturas orofaciais; a mastigação quanto ao tipo, tempo e número de ciclos mastigatórios, e a deglutição em indivíduos adultos usuários de prótese dentária removível e com dentição natural não se diferenciam. Os grupos se diferenciam quanto ao corte do alimento, onde a presença e tempo de uso da prótese parecem interferir nessa variável.

\section{ABSTRACT}

Purpose: to check mastication characteristics in individuals using removable dental prosthesis, analyzing probable interference factors associated to functional mastication. Methods: fifty-three individuals of both genders, between 42 and 67year old, were divided in two groups: thirty-two individuals, users of total stable prosthesis dentures and/or partially stable removable prosthesis (G1); and twentyone individuals with natural dentition (G2). the following exclusion criteria were used: neurological or cognitive deficiency, dental facial deformities, temporomandibular disorders and any mouth injuries which could interfere in mastication. Evaluation was done with salt bread, using habitual mastication. The analyses focused on: oral structures and muscle characteristics; type of cutting, maintenance of labial sealing; length of mastication time, number of masticatory cycles, and type of mastication: uni or bilateral, considering unilateral predominance in more than $66 \%$ of the cycles on just one particular side. Results: alterations were noted as for the characteristics of the masseter muscle and type of food cutting for G1, with significant statistical differences when compared to G2. All other orofacial structures: lips, tongue, cheeks, mentual, and position of lips during mastication proved to be normal for both groups. There was no significant difference between the groups regarding jaw movements; type, time, and number of masticatory cycles and deglutition characterization. Conclusion: mastication in removable prosthesis users is characterized by alterations in the form of cutting food, undefined type of mastication, and satisfactory lips posture. The average time and the number of masticatory cycles are similar to those obtained for individuals with natural dentitions.

KEYWORDS: Dental Prosthesis; Denture, Partial, Removable; Mastication

\section{REFERÊNCIAS}

1. Cunha CC, Felício CM, Bataglion C. Condições miofuncionais orais em usuários de próteses totais. Pró-Fono. 1999; 11(1):21-6.

2. Fazito LT, Perim JV, Di Ninno CQMS. Comparação das queixas alimentares de idosos com e sem prótese dentária. Rev CEFAC. 2004; 6(2):143-50.

3. Veyrune JL, Mioche L. Complete denture wearers: electromyography of mastication and texture perception whilst eating meat. Eur J Oral Sci. 2000; 108(2):83-92.

4. Miyaura K, Morita M, Matsuka Y, Yamashita A, Watanabe T. Rehabilitation of biting abilities in patients with different types of dental prostheses. J Oral Rehabil. 2000; 27(12):1073-6.

5. Kapur KK, Soman SD. Masticatory performance and efficiency in denture wearers. 1964. J Prosthet Dent. 2006; 95(6):407-11.

6. Corpas LS. Avaliação da função mastigatória associada à força de mordida e percepção oral em indivíduos portadores de prótese total [dissertação]. Bauru (SP): Universidade de São Paulo; 2005.
7. Prado MMS, Borges TF, Prado CJ, Gomes VL, Neves FD. Função mastigatória de indivíduos reabilitados com próteses totais mucoso suportadas. Pesq Bras Odontoped Clin Integr. 2006; 6(3):259-66.

8. Yven C, Bonnet L, Cormier D, Monier S, Mioche L. Impaired mastication modifies the dynamics of bolus formation. Eur J Oral Sci. 2006; 114(3):184-90.

9. Piancino MG, Farina D, Talpone F, Castroflorio T, Gassino G, Margarino V, Bracco P. Surface EMG of jaw-elevator muscles and chewing pattern in complete denture wearers. J Oral Rehabil. 2005; 32(12):863-70.

10. Felício CM, Fernandes RSM, Silva MAMR. Investigação da relação entre o tipo mastigatório e o limite de movimento lateral da mandíbula. Rev Soc Bras Fonoaudiol. 2005; 10(2):71-6.

11. Felício CM, Cunha CC. Relações entre condições miofuncionais orais e adaptação de próteses totais. Rev Bras Prót Clin Lab. 2005; 7(36):195-202.

12. Kalil MTAC, Cavalcanti RVA, Bianchini EMG. Protocolo de Avaliação Miofuncional Orofacial para Usuários de Próteses Dentárias. In: Anais 
Suplemento especial da Revista da Sociedade Brasileira de Fonoaudiologia. 160 Congresso Brasileiro de Fonoaudiologia; 2008 Set 24-27; Campos do Jordão, São Paulo. Disponível em: URL: http://www.sbfa.org.br/portal/anais2008

13. Bianchini EMG. Avaliação fonoaudiológica da motricidade oral. In: Bianchini EMG. Articulação temporomandibular: implicações, limitações e possibilidades fonoaudiológicas. Carapicuíba: PróFono; 2000. p.191-253.

14. Whitaker ME. Função Mastigatória: proposta de protocolo de avaliação clínica [dissertação]. Bauru (SP): Universidade de São Paulo; 2005.

15. Bianchini EMG. Mastigação e ATM Avaliação e Terapia. In: Marchesan IQ. Fundamentos em fonoaudiologia: aspectos clínicos da motricidade oral. 2. ed. Rio de Janeiro: Guanabara Koogan; 2005. p. 45-57.

16. César SR, Bommarito S, Ramos LR. Avaliação da mastigação de idosos: um estudo sobre adaptação da prótese dentária superior e inferior e a função mastigatória. Rev Fono Atual. 2004; 7(29):13-8.

17. Melo TM, Arrais RD, Genaro KF. Duração da mastigação de alimentos com diferentes consistências. Rev Soc Bras Fonoaudiol. 2006; 11(3):170-4.

18. Mbodj EB, Ngom PI, Seck MT, Ndiaye C, Dieng L, Touré SN, et al. Study of the characteristics of the food bolus in elderly subjects whit complete dentures. Odontostomatol Trop. 2007; 30(119):11-6.
19. Berretin-Felix G, Genaro KF, Trindade IEK, Trindade Júnior AS. Masticatory function in temporomandibular dysfunction patients: electromyographic evaluation. J Appl Oral Sci. 2005; 13(4):360-5.

20. Ribeiro RA, Mollo Júnior FA, Pinelli LAP, Arioli Júnior JN, Ricci WA. Prevalência de disfunção craniomandibular em pacientes portadores de próteses totais duplas e pacientes dentados naturais. Cienc Odontol Bras. 2002; 5(3):85-9.

21. Ribeiro RA, Mollo Júnior FA, Arioli Júnior JN, PinelliLAP. Sintomas de disfunçãocraniomandibular. RGO. 2003; 51(2):123-6.

22. Leles CR, Compagnoni MA, Souza RF, Barbosa DB. Kinesiographic study of mandibular movements during functinal adaptation to complete dentures. J Appl Oral Sci. 2003; 11(4):311-8.

23. Tagliaro ML, Calvi CL, Chiappetta ALML. A fase de incisão no processo da mastigação: enfoque clínico. Rev CEFAC. 2004; 6(1):24-8.

24. Duarte LIM. Relação entre maloclusão e mastigação. Rev Soc Bras Fonoaudiol. 2001; 6(1):46-51.

25. Mizumori $T$, Tsubakimoto $T$, Iwasaki $M$, Nakamura T. Masticatory laterality: evaluation and influence of food texture. J Oral Rehabil. 2003; 30(10):995-9.

26. Nissan J, Gross MD, Shifman A, Tzadok L, Assif D. Chewing side preference as a type of hemispheric laterality. J Oral Rehabil. 2004; 31(5):412-6.

RECEBIDO EM: 12/05/2008

ACEITO EM: 29/10/2008

Endereço para correspondência:

Av. Resplendor, 95/701

Vila Velha - ES

CEP: 29101-500

Tel: (27) 8824-0510

Fax: (27) 3329-8039

E-mail: revacavalcanti@gmail.com 